\title{
Low-Frequency Oscillation in Power System
}

\section{Abhinav Pathak, Ratnesh Gupta}

\begin{abstract}
Low-frequency oscillation can collapse the stability of the power system, which is considered to be one of the most significant challenges to a power system engineer. In earlier decades modal analysis was carried out for identifying lowfrequency oscillation modes, which have various drawbacks. In the present era, with the application of the Phasor measurement unit \& various signal processing techniques, identification of lowfrequency oscillation is being carried out with accuracy to an extent. This paper provides a survey of recent research and development in the field of identification of low-frequency oscillation by different signal processing techniques. It is expected that this literature survey will provide researchers with some future direction in finding relevant references and developing suitable techniques for low-frequency oscillation detection in the interconnected power system.
\end{abstract}

Keywords: Inter-area modes, Low-frequency oscillation, Phasor measurement unit, Power system.

\section{INTRODUCTION}

Small signal stability is the capacity of a power system to maintain synchronization when there are small disturbances, which occur continuously in the system, due to small changes in load and generation. The disturbances are expected to be small enough to enable the linearization of system equations for the purpose of analysis [1]. To analyze a non-linear dynamic system, modal analysis is a notable tool to understand the small-signal stability of the power system. In the modal analysis, the non-linear differential equations of the system should be linearized at any given operating point to obtain eigenvalues. Then the obtained eigenvalues are used to determine oscillation modes and their shape. The dynamic parameters of the power system can be determined using the model-based technique at a certain operating point. In a power system, the operating point may vary with respect to time due to variation in load-generation, so it becomes difficult to obtain precise parameters for the design of the exact power system[1-2].

In the power system, the utilization of Phasor Measurements Units (PMUs) has increased recently. Hence many researchers are focused to develop a measurementbased method with the application of PMU and are practically implemented in the power system [2, 3]. The PMUs can provide a common reference to time-tagged voltage and current phasors. In comparison with traditional data monitoring and acquisition systems, the data is available at a higher rate at the control centre, thus allowing a broad spectrum of applications for electric power systems.

By means of an appropriate algorithm, the time-series data is to be analyzed to provide relevant information to the operator [3].

Revised Manuscript Received on January 05, 2020.

Abhinav Pathak DAVV, School of Instrumentation Indore M.P; Medi-Caps University, Electrical Engineering Department Indore M.P

Ratnesh Gupta DAVV, School of Instrumentation Indore M.P
For a power system, there are two modes of operation i.e. normal or ambient operation and ring-down or transient operation. Due to the sudden variation of load in the power system, the modes get excited during ambient operation leading to a transient state of operation. In addition, the transient mode of operation also refers to the power system's non-linear behaviour in response to large disruptions, and the low-frequency ring-down oscillations are observed in those cases. The power system may face low-frequency oscillation problems because of randomly varying load conditions or system disturbances. The low-frequency oscillation (inter-area) lies within the range of $0.1-1.0 \mathrm{~Hz}$. The oscillations must be reduced sufficiently to improve the stability conditions of the power system. If sufficient damping is not present in the system, instability may occur which collapses system operations in the power system. So, to identify low-frequency oscillations, several techniques are developed by the researcher.

There exist several ring-down based algorithms for mode identification (damping-ratio and frequency). For identification of the model parameters during ring-down oscillations in the power system, the Prony algorithm is treated as a reference algorithm. The challenge of extracting dominating modes from the signal in a wide range of fictitious modes generated by the algorithm is a significant constraint of the Prony algorithm for real-time applications.

With the growing introduction of renewable energy sources into the power system, the damping of the critical modes of the system and system inertia is reduced. However, this paper focuses mainly on identifying dominant low-frequency modes in the power system by small-signal stability and mainly algorithm derived from Prony analysis.

The remaining portion of the article is structured in the following way. Section II outlines the general preliminaries of small-signal stability in the power system. Section III provides a review of the literature on the detection of dominant low-frequency modes in the power system and Section IV provides the conclusion.

\section{ANALYSIS OF SMALL SIGNAL STABILITY: BASIC PRELIMINARIES}

The load requirement in the modern power system is rapidly increasing, which creates stress and stability problems in the system.

System operating conditions may create instability problems in the power system and are difficult to predict in advance. Small-signal stability analysis is based on the derivation of a linear model of the nonlinear system model around a particular operating condition. 
The eigenvalues $(\lambda)$ can be computed to analyze the stability of the linear model using state matrix $A$. The right eigenvector $(\varphi)$ in addition to the left eigenvector $(\psi)$ is considered as the participation of each system state for a specific eigenvalue. The system matrix eigenvalue and the related eigenvectors are given by the following equation as: $\mathrm{A} \Phi_{\mathrm{i}}=\lambda_{\mathrm{i}} \Phi_{\mathrm{i}}$

$\varphi_{\mathrm{i}} \mathrm{A}=\lambda_{\mathrm{i}} \varphi_{\mathrm{i}}$

In the case of a complex eigenvalue corresponding to the system oscillatory mode, the oscillation frequency in $\mathrm{Hz}$ and the damping ratio $(\xi)$ are given by the following expression as:

$$
\begin{aligned}
& \lambda_{\mathrm{i}}=\sigma_{\mathrm{i}} \pm j \omega_{\mathrm{i}} \\
& f_{i}=\frac{\omega_{\mathrm{i}}}{2 \pi} \\
& \xi_{i}=-\frac{\sigma_{i}}{\sqrt{\sigma_{i}^{2}+\omega_{i}^{2}}}
\end{aligned}
$$

Eq. (5) gives the damping ratio, which is associated with the real part of the eigenvalue, which defines the rate at which the amplitude of oscillation decreases. For the purpose of stability, if the location of poles (eigenvalue) is in the left half of the complex plane, then the system is said to be stable. If the poles (eigenvalue) are located on the right half or in the repeated form on the imaginary axis then the system becomes unstable.

\section{REVIEW OF LITERATURE ON DOMINANT LOW-FREQUENCY MODE IDENTIFICATION IN POWER SYSTEM}

Identifying low-frequency oscillations in the power system is an essential part of improving stability under varying load and generation conditions. Identifying accurately true modes present in a signal is of high importance to maintain stability, if true modes are not identified accurately or if some fictitious modes are identified by processing algorithm it may lead to improper control action which can affect the operation and stability of the power system and may also lead to the collapse of the system. Researchers have developed several different algorithms to identify low-frequency oscillations by calculating specific parameters from the input signals. In this section, recently developed methods for identifying parameters in the input signal are analyzed and described as follows.

In [3], an algorithm associated with the multi-Prony model was developed to detect the dominant low-frequency modes in the interconnected power system. The proposed technique is based on the fact that the actual modes present in the signals appear continuously, regardless of the order of the Prony algorithm, and true modes are retrieved with a sorting process. This paper suggests an improved Prony algorithm based on a shrinking window concept is proposed in which several sub-windows are created within the main window and the true modes are extracted by comparing Euclidean distance between individual modes in the complex plane. The results of the proposed algorithm are verified by considering different possible variations in synthetic signal and on a 16generator 68-bus system. On the basis of results shown in this research paper, it is concluded that the algorithm is capable of determining actual modes present in the input signal even in the presence of noise and specific PMU recording levels.

In 1991, basic research to examine the inter-area oscillatory modes in the power system was described in [4]. The purpose of the analysis was to define the fundamental properties of low-frequency oscillation in the large power system. The developed technique was utilized for modelling reduced order systems in addition to adjusting and designing procedures. In this research, the author has considered two area systems for the identification of oscillatory modes by examining signal stability and transient stability. The variation due to tie-line impedance, excitation system in addition to load characteristics on inter-area oscillation was studied in this research. This research work has great significance for power system engineers to understand the nature of inter-area oscillatory modes in the power system.

In 2017, low-frequency oscillatory modes were identified in the power system using an algorithm based on EMO ESPRIT is described in [5]. ESPRIT method is simple but provides incorrect result if the modes present in the signal is closely spaced or less in number, in order to overcome this problem researcher in this paper has developed novel model order estimation technique so that algorithm can identify true modes accurately even in presence of noise. The researcher develops an exact model order algorithm in this paper to pre-estimate the exact number of modes. The idea behind this algorithm was that the signal and noise sub-space have significantly different eigenvalues. The above principle was used to effectively differentiate the autocorrelation matrix of signal and noise subspace. In this study, the exact model order estimation algorithm and ESPRIT were combined to analyze lowfrequency oscillations. Different combinations of synthetic signals with various noise levels and different PMU reporting rates are used in this research work to check the performance of the developed method. The developed technique validity was confirmed on the probe test data collected from WECC. From the results reported by the researcher, it can be inferred that precise measurement of the damping and frequency of the signal even in presence of noise can be obtained from the proposed method, without producing any fictitious mode.

The author in [6] developed a novel oscillation computation technique associated with an improved Prony algorithm by (SVD-DDM) singular value decomposition differential method and a novel monitoring process for tracking dominant oscillation. In earlier research, several Prony based methods are proposed by researchers but the most crucial problem of accurate model order estimation is still a major drawback of this method so the author in this paper has made attempt to overcome this problem. For the order estimation of the Prony algorithm, the SVD-DDM approach is proposed in this research paper. 
In order to eliminate the noise effect, another parameter optimization approach based on the interior point method is developed. The major contribution of the paper lies in the novel model order determination algorithm and parameter optimization method; the actual PMU data from China's electric grid and EPRI-36 system was used to evaluate the performance of the proposed method.

In [7] the author provides a comparative analysis of two modal identification techniques i.e. Prony analysis \& Hilbert transform technique. The author had contrasted the Prony algorithm with the Hilbert transform in terms of performance measures such as measurement noise, accuracy, and capability to differentiate between two similar modes. The Hilbert transform and the empirical mode decomposition (EMD) were able to identify oscillations in the non-stationary signal, while the Prony method is for the stationary signal. There exist several structural differences between both techniques. Hilbert transform isn't able to distinguish two modes separately if the difference between modes (damping ratio or frequency) is large. The two approaches analyzed provide very different information and it will be beneficial if the two methods are used in a complementary manner [7].

The author in [8] has developed a hybrid technique for the calculation of low-frequency oscillation for an interconnected power system. With the application of wide-area measurement, the researcher has developed a hybrid method that consists of four different algorithms i.e. Discrete Fourier transform, finite-impulse response (FIR) filter, prototype algorithm, and the least square error method. The Discrete Fourier transform is the first algorithm used to begin the mechanism of estimating the low-frequency oscillations. Then the second is the finite-impulse response (FIR) filter used to filter undesirable components, and the window correction factor is used to remove the FIR window filter ill effect. The prototype algorithm is the third algorithm, it was used to compute parameters for identifying low-frequency oscillations. The least-square error is the final method, it was used to enhance the accuracy in the computation period through variation of the window side. The proposed method is validated by field test data of PMU from Taipower system and verified by simulation results from consideration of two machine transmission system in MATLAB/Simulink [8].

In 2016, an algorithm based on the Zolotarev Polynomial filter bank was proposed in [9] to compute the inter-area oscillations. Zolotarev Polynomial Filter Bank (ZPBFB) is used for decompositions of PMU signals into mono components. The modal frequency and damping were obtained from the mono component signal with help of Eigen realization algorithm (ERA) [9]. The developed method was tested on the 68 bus network (16 machines) and two area test system. Additionally, cosine modulated filter bank (CMFB) was tested with the above-mentioned system and compared the ZPBFB. The developed method was validated with real-time signals collected from a wide-area measurement system (WAMS) at three locations on 30 November 2011 in India for estimation of signal parameters i.e. damping ratio and frequency.

The author in [10] have developed Ensemble Empirical Mode Decomposition (EEMD) and utilized the well-known Prony algorithm for computing the parameters of lowfrequency oscillation. The original signal is decomposed on the basis of the frequency range size of the signal, then the intrinsic mode function (IMF) is obtained and the IMF that contains the dominant mode is determined through the signal's weight of energy weight and ultimately the IMF will be examined using the Prony algorithm to determine modal parameters of the low-frequency signal [10]. The approach proposed is verified to determine low-frequency oscillation modes in presence of noise signal for two systems i.e. two-area four machine system and for the analog signal of the Sichuan power grid.

For accurate detection of inter-area modes present in the signal initially, model-order is to be predetermined which is very important to get accurate parameters of the signal i.e. frequency and damping ratio of the signal from signal processing algorithm. If model-order isn't calculated accurately then the result (modes) obtained from the processing algorithm will not be the one that is actually present in the signal, if true modes are not identified accurately or if some fictitious modes are identified by processing algorithm it may lead to improper control action. In [11] the author has developed three novel model order determination algorithm to correctly identify the exact number of modes present in the signal. The proposed algorithm is based on the basis of change observed in the eigenvalues obtained from the auto-correlation matrix of the signal. The performance of three algorithms is verified on the synthetic signal with the different number of modes at different noise levels, on basis of computation time required, and on the field data from generating station. The performance of three algorithms varies based on the complexity, nature of signal and noise level. If the performance in terms of accuracy for a particular algorithm is satisfactory at higher noise level than the computation time of algorithm increases, in the same way, if the computation time required for a particular algorithm is lesser than its accuracy at higher noise level isn't good. Depending upon the requirement in terms of accuracy and computation time one has to wisely decide a suitable algorithm among three algorithms for calculation of the number of modes present in the signal.

All the parametric methods available in the literature for identifying inter-area modes require the correct model order of the system to get true modes present in the signal. Fourier transform-based methods are simple to implement but they are not capable to identify true modes if the modes are closely spaced, also they can't provide damping ratio directly. In [12] an algorithm derived from Hankel's least square technique (HLS) and Fourier transform is proposed to identify true modes of oscillation. Fourier transform is used to identify the model order of the system and further HLS technique is applied to identify parameters of the signal. The proposed algorithm is validated by the author on the simulated synthetic signal with the different noise level and on real-time data, also the performance is compared with two existing techniques available in the literature, the result of the proposed algorithm is more accurate as compared with respect to existing techniques. 


\section{Low-Frequency Oscillation in Power System}

In the past decades already several techniques are discussed by different authors for accurate detection of interarea modes present in the signal. In [13] the author has proposed a new technique in which initially noise present in the signal is removed by means of stationary wavelet transform technique which is better in comparison to other noise filtering techniques existing in literature, this waveletbased method provides better-filtered signal up to $15 \mathrm{~dB}$ SNR. The author in this research further develops an exact model order algorithm in this paper to pre-estimate the exact number of modes. The idea behind this algorithm was that the signal and noise sub-space have significantly different eigenvalues. The above principle was used to effectively differentiate the auto-correlation matrix of noise and signal subspace. For the calculation of signal parameters (damping ratio and frequency) the author has utilized a stochastic subspace-based identification (SSBI) method after filtering noise content and identifying the number of modes present in the signal. These SSBI methods are gaining popularity among power system engineers as it can handle large data and dynamic changes happening in the system. But the limitations with SSBI method are that its accuracy and performance are dependent on measurement noise and accurate model order identification, but in this research both the constraints are overcome by proper filtering of the signal and by accurate determination of model order. The proposed algorithm is validated by the author on the simulated synthetic signal with the different noise level and on realtime PMU data obtained from two systems, also the performance is compared with two existing techniques available in the literature, the result of the proposed algorithm is more accurate as compared with respect to existing techniques.

In [14] an algorithm derived from neural network and Fourier transform is proposed to identify true modes of oscillation. The author in this work applied Fourier transform to identify the model order of the system and further a neural network-based algorithm is applied to identify parameters of the signal. The proposed algorithm is validated by the author on the simulated synthetic signal with the different noise levels and on real-time data obtained from the power grid of China.

\section{CONCLUSION}

On the basis of the review, it can be concluded that there are several existing techniques in literature for identifying low-frequency oscillation parameters in the power system. It was found that the Prony algorithm was used as a benchmark algorithm and most widely accepted among power system engineers. But the Prony algorithm has some notable limitations as it was highly dependent on model order i.e. if the model order is high then the Prony algorithm produces a number of fictitious modes and it is very challenging to identify true dominant modes in an input signal. Moreover, the algorithm may not detect some of the dominant oscillation modes contained in the signal if the model order is set too low. Another limitation with the Prony algorithm is its dependence on measurement noise the result of the Prony algorithm is deteriorated in the presence of noise, in the same way, other methods available in the literature have their own pros and cons.

It is very essential to identify the exact parameter of lowfrequency oscillation, to achieve and maintain the stability system and for performing appropriate control action whenever required in a power system. So there is a future scope to develop a novel algorithm that is simple and is accurate for identifying low-frequency modes in the power system.

\section{REFERENCES}

1. Kundur P, Balu NJ, Lauby MG. Power system stability and control, vol. 7. NewYork: McGraw-Hill; 1994.

2. Jiang T,Bai L, Li G, Jia H,Hu Q, Yuan H : 'Estimating inter-area dominant oscillation mode in bulk power grid using multi-channel continuous wavelet transform', Journal of Mod. Power Syst. Clean Energy, 2016, 4 (3), pp (394-405).

3. Wadduwage D.P, Annakkage U.D, Narendra K : 'Identification of dominant low-frequency modes in ring-down oscillations using multiple Prony models', IET Gener. Transm. Distrib., 2015, 9,(15) pp(2206-2214)

4. Klein M., Rogers G.J, Kundur P: 'A Fundamental study of inter-area oscillations in power systems', Transactions on Power Systems, 1991,6,(3),pp(914-921).

5. Philip J.G, Jain T: 'Analysis of low frequency oscillations in powe system using EMO ESPRIT', Electrical Power and Energy Systems, 2018, 95, pp (499-506).

6. Xia X, Li C, Ni W: 'Dominant low-frequency oscillation modes tracking and parameter optimisation of electrical power system using modified Prony method', IET Gener. Transm. Distrib., 2017,11 (17), pp(4358-4364)

7. Browne T.J, Vittal V, Heydt G.J, Messina A.R: 'A Comparative Assessment of Two Techniques for Modal Identification From Power System Measurements', IEEE TRANSACTIONS ON POWER SYSTEMS, 2008, 23(3), pp (1408-1415).

8. Yang J-Z, Liu C-W, Wu W-G: 'A Hybrid Method for the Estimation of Power System Low-Frequency Oscillation Parameters', IEEE TRANSACTIONS ON POWER SYSTEMS, 2007, 22 (4) pp(2115 2113).

9. Mandadi K Kumar B.K: 'Identification of Inter-Area Oscillation Using Zolotarev Polynomial Based Filter Bank With Eigen Realization Algorithm' ,IEEE TRANSACTIONS ON POWER SYSTEMS,2016,31 (6) pp (4650-4659).

10. Gao J,Guo C: 'Method of Modal Parameter Identification for Power System Low Frequency Oscillation Based on EEMD and Prony Algorithm', Automatization,2015,6,pp(76-83).

11. Piyush Warhad Pandea, Bandi Ravi Kumara, Saikat Chakrabartia Suresh Chandra Srivastavaa , Subrata Sarkarb , Tarun Sharma 'Model order estimation methods for low frequency oscillations in power systems', Electrical Power and Energy Systems,2019,115.

12. Joice G. Philip, Trapti Jain : 'Estimation of modal parameters of low frequency oscillations in power system using Hankels total least square method', 2018 IEEE Innovative Smart Grid Technologies Asia (ISGT Asia) pp (764-769).

13. Joice G. Philip, Trapti Jain: 'An improved Stochastic Subspace Identification based estimation of low-frequency modes in power system using synchrophasors ', 2019, pp (495-503).

14. Zhongting Shen, Renjie Ding: 'A Novel Neural Network Approach for Power System Low Frequency Oscillation Mode Identification', 2019 IEEE International Symposium on Circuits and Systems (ISCAS)

\section{AUTHOR PROFILE}

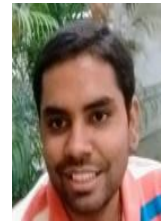

Abhinav Pathak is working as Assistant Professor at Medi-Caps University Indore in the Electrical Engineering Department. $\mathrm{He}$ is pursuing Ph.D. from School of Instrumentation, DAVV Indore. His current area of research is signal processing application to the power system for the identification of inter-area modes. E-mail id: abhinavgsits7@gmail.com / abhinavp436@gmail.com

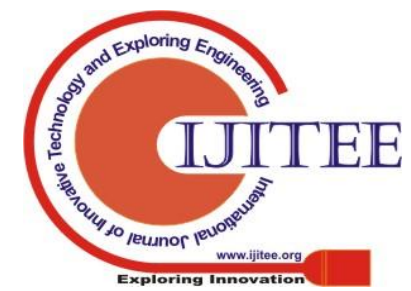


Ratnesh Gupta is working as Professor and Head of Department at School of Instrumentation, DAVV Indore. His area of research includes instrumentation, control system, signal processing, etc. He has vast research as well as teaching experience and guided several M. Tech and Ph.D. scholars. He has published several research papers in reputed journals.

mail id: ratneshg@hotmail.com 